\title{
Pulmonary tuberculosis manifesting as 'tree-in-bud' opacities on chest radiography
}

\author{
RUMi R. KHAJOTIA ${ }^{1}$, SANGEETHA POOVANESWARAN ${ }^{2}$
}

Keywords: Pulmonary tuberculosis, Tree-in-Bud pattern, HRCT chest scan

\section{Background}

The 'tree-in-bud' pattern is an appearance typically seen on HRCT or thin-section computed tomography (CT) chest scan. It is characterized by small centrilobular nodules of soft-tissue attenuation connected to multiple branching linear structures of similar caliber which originate from a single stalk [1-3]. Initially described in cases of endobronchial spread of Mycobacterium tuberculosis [1], it has subsequently been observed as a manifestation of a variety of entities, including idiopathic disorders (obliterative bronchiolitis and panbronchiolitis), infections (bacterial, viral, fungal and parasitic) involving peripheral airways, immunologic disorders, connective tissue disorders, aspiration, inhalation, congenital disorders and pulmonary intravascular tumor emboli (carcinomatous endarteritis). In patients with pulmonary tuberculosis, the tree-in-bud pattern is believed to be a characteristic CT feature of active endobronchial spread and can be found in $72 \%$ of patients with active disease [2]. We report here, the case of a young woman with breast carcinoma who developed pulmonary tuberculosis while on chemotherapy and whose HRCT chest scan showed characteristic 'treein-bud' opacities.

\section{Case presentation}

A 42-year-old female presented with a lump in her left breast. There were no other significant symptoms. On examination, a $4 \mathrm{~cm}$ lump was palpable in the upper outer quadrant of the left breast. Mammography confirmed the presence of a $4 \mathrm{~cm}$ mass with spiculate margins and distorted architecture. Core biopsy was performed which confirmed the presence of a grade II infiltrating ductal carcinoma. A left-sided modified radical mastectomy was performed with level II axillary clearance. Nine out of sixteen nodes from the axillary clearance showed presence of metastatic deposits. She was subsequently started on 3 weekly cycles of chemotherapy which included 5-flurouracil, epirubicin and cyclophosphamide. Five months later, the patient presented with dry cough, low-grade fever with an evening rise of temperature, generalised weakness and loss of appetite. She had lost 4 kilograms of weight in the last 3 months. On examination, a few coarse crepitations were heard over the mid-zone of her right lung. Chest radiograph was unremarkable and the total white cell counts were normal $(7.6 \times 109$ cells/ L). An HRCT chest scan was done. It showed the presence of peripheral, small, centrilobular, well-defined nodules of soft-tissue attenuation connected to linear, branching opacities that $\overline{1 \text { Internal Medicine and Pulm- }}$ onology, International Medical University Clinical School, Malaysia

2Internal Medicine, International Medical University Clinical School, Malaysia

CORRESPONDING AUTHOR: Rumi R. Khajotia

Internal Medicine and Pulmonology, International Medical University Clinical School, Malaysia rumi@imu.edu.my; kkhajotia94@yahoo.com

Funding: This research received no specific grant from any funding agency in the public, commercial, or not-for-profit sectors. 
had more than one contiguous branching site originating from a single stalk, resembling a typical 'tree-in-bud' pattern (Figure. 1, 2, 3\&4). No mediastinal or hilar gland enlargement was noted and no other lesions were seen. A differential diagnosis of pulmonary tuberculosis, pulmonary infections (bacterial, viral, fungal and parasitic) involving peripheral airways and pulmonary intravascular tumor emboli (carcinomatous endarteritis) was considered.

Initial examination of three earlymorning sputum samples was negative for acid-fast bacilli (AFB) and therefore pulmonary intravascular tumor emboli were considered a likely possibility. However, subsequently, induced sputum samples were collected using 3\% hypertonic saline solution delivered by an ultrasonic nebulizer (Devilbiss Ultraneb 99, PA, USA). Three early morning induced sputum samples were collected on consecutive days. On smear examination, the samples were positive $(+++)$ for AFB. Subsequently, culture of the sputum samples confirmed presence of live AFB. The sputum samples were negative for malignant cells and bacterial culture. Tests for rheumatoid arthritis and Sjogrens syndrome too were negative.

In view of her history and investigative findings, a diagnosis of pulmonary tuberculosis manifesting as 'tree-in-bud' opacities was made and the patient was started on anti-tuberculous treatment. After 2 months of treatment, the cough and fever had subsided, her appetite had improved and she had gained $3 \mathrm{kgs}$ of weight.

\section{Discussion}
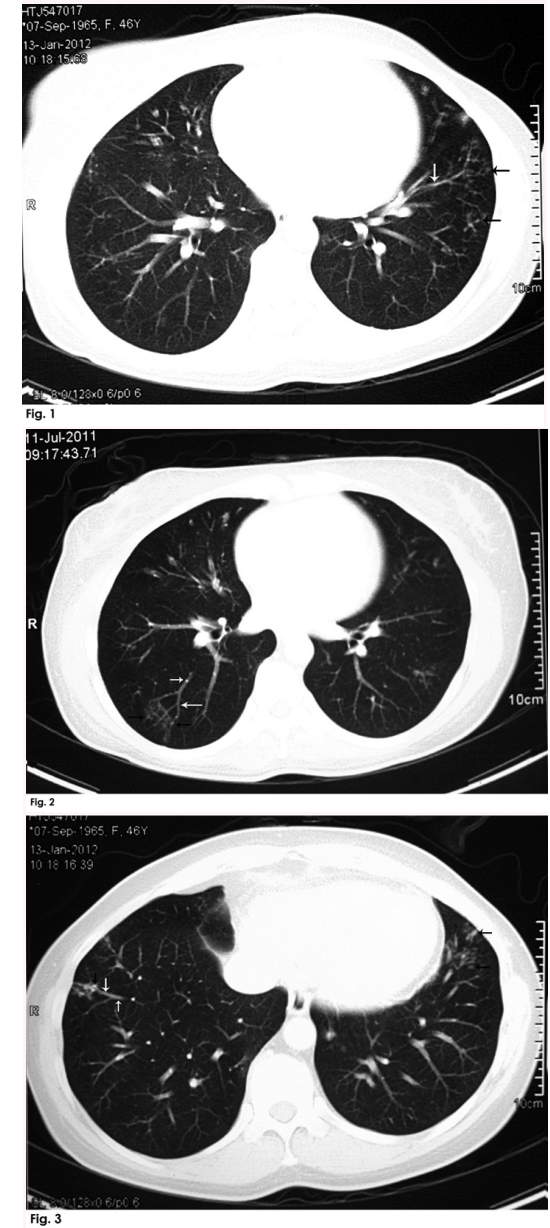

Figure 1,2\&3. 'Tree-in-bud' pattern seen in both lower lobes. Centrilobular, well-defined nodules of soft-tissue attenuation are seen (black arrows), connected to linear, branching opacities (large white arrows) which originate from a single stalk (small white arrows)

Pulmonary tuberculosis is an endemic disease with a rising global incidence, where prompt diagnosis is crucial in instituting early treatment. Hence, it is vitally important to be well-acquainted with the various clinical and radiological manifestations of this disease, so as to make the diagnosis early in the disease process.

Pulmoanry tuberculosis is known to have a wide spectrum of clinical and


Fig. $4 \mathrm{c}$

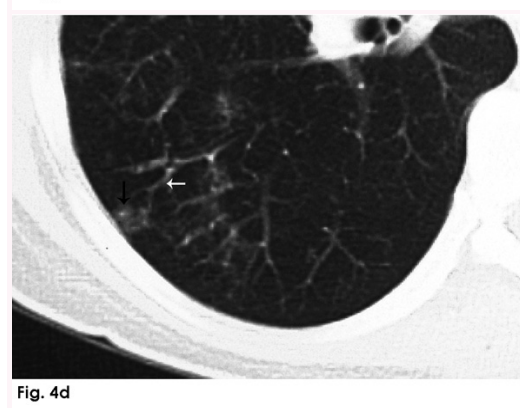

Figure 4a,b,c\&d. Magnified HRCT images of both lower lobes obtained using 1-mm collimation and lung window settings show multiple centrilobular nodules (black arrows) and branching lines (white arrows), depicting the 'tree-inbud' appearance. 
radiological manifestations and is also known to mimick other disease processess. An important radiographic feature of pulmonary tuberculosis is the "tree-inbud' pattern which is a valuable indicator of active tuberculosis in patients who have the underlying clinical symptoms and signs.

The 'tree-in-bud' sign is usually seen on HRCT or thin-section CT images of the lung. It has occasionally been reported in patients with endobronchial spread of Mycobacterium tuberculosis and is believed to be highly suggestive of active tuberculosis [1,3]. It consists of peripheral, small, centrilobular nodules of soft-tissue attenuation connected to linear branching opacities that have more than one contiguous branching site which originate from a single stalk. This is believed to be due to caseous material plugging the small airways. The impaction is composed of secretions or fibrotic material manifesting as 2 to $4 \mathrm{~mm}$ nodular and linear branching centrilobular opacities on an HRCT chest scan. The terminal tufts of the 'tree-in-bud' pattern represent inflammation with caseous material in the respiratory bronchioles and alveolar ducts, whereas the stalks represent caseous material within the terminal bronchioles.

Diagnosing pulmonary tuberculosis can sometimes prove to be difficult, since it is largely dependent on the quality of the sputum sample tested for AFB. Consequently, studies have shown that AFB are found in sputum samples in only a limited number of patients $(20 \%$ to $55 \%$ ) with active disease [4]. Compounding this is the fact that chest radiography may also be indeterminate in a large number of patients. Hence, in such situations, HRCT chest scan findings prove to be very useful in detecting early tuberculous infection when chest radiographic findings are inconclusive [5]. Im et al. [2] evaluated the HRCT findings in 41 patients newly diagnosed with active pulmonary tuberculosis. They found centrilobular lesions appearing as nodules or as branching linear structures (95\%), bronchial wall thickening (73\%), 'tree-in-bud appearance' (71\%), poorly defined nodules $(61 \%)$, cavities $(51 \%)$, and lobular consolidation (41\%). The endobronchial spread of mycobacterium tuberculosis represents a chronic granulomatous infection in which active organisms spread via the airways after caseous necrosis of the bronchial wall. Hence, one of the earliest appearances is that of a 'tree-in-bud' pattern consisting of 2 to $4 \mathrm{~mm}$ centrilobular nodules and branching linear structures of similar calibre originating from a single stalk.

Consequently, though chest radiography remains the first diagnostic option for evaluating patients with pulmonary tuberculosis; in recent years, HRCT chest scan has been found to be superior to both chest radiography and standard $\mathrm{CT}$ in the assessment of parenchymal lung diseases [3]. Micro-nodules and the 'tree-in-bud' appearance are characteristic HRCT features in tuberculosis patients with recent infection, findings not usually seen on conventional chest radiography.

\section{Conclusion}

This case report highlights the fact that a high-index of clinical suspicion when accompanied by the typical 'tree-in- bud' patterning on HRCT chest scan is useful in suggesting the diagnosis of active pulmonary tuberculosis early in the disease process, thereby avoiding delay in initiation of treatment. Consequently, knowledge of this important radiographic pattern would be of considerable importance to both family physicians and consultants who encounter pulmonary tuberculosis frequently in the course of their busy medical practice.

\section{Consent}

Consent has been obtained from the patient for purpose of publishing the case report.

\section{Competing interests}

The author(s) declare that they have no competing interests.

\section{References}

1. Eisenhuber E. The tree-in-bud sign. Radiol 2002;222:771-2.

2. Im JG, Itoh H, Shim YS, J H, Ahn J, Han M C, Noma S. Pulmonary tuberculosis: CT findings-early active disease and sequential change with antituberculous therapy. Radiol1993;186:653-60.

3. Hatipoğlu ON, Osma E, Manisali M, Uçan ES, Balci P, Akkoçlu A. High resolution computed tomographic findings in pulmonary tuberculosis. Thorax 1996;51:397-402.

4. Hong YP, Kwon DW, Kim SJ. Tuberculosis in Korea. In: Hong YP, editor. Management of tuberculosis. Seoul: Ministry of Health and Social Affairs, Korean Academy of Tuberculosis; 1990. pp. 3-4.

5. Lee KS, Im JG. CT in adults with tuberculosis of the chest: characteristic findings and role in management. AJR Am J Roentgenol 1995;164:1361-7. 